\title{
Estado actual del condrosarcoma en un centro de referencia
}

\author{
Current status of chondrosarcoma in a reference center \\ Clara-Altamirano MA, * García-Ortega DY, Núñez-González M, \\ Caro-Sánchez CHS, "Espejo-Sánchez G," Martínez-Said H, ${ }^{\ddagger}$ Cuéllar-Hübbe M** \\ Instituto Nacional de Cancerología, CDMX.
}

\begin{abstract}
RESUMEN. Introducción: El condrosarcoma es el segundo tumor óseo maligno primario más frecuente, se caracteriza por la formación de matriz condroide, es más común en edades avanzadas y su pronóstico guarda relación directa con el subtipo histológico y el grado tumoral. El objetivo de este artículo es presentar el perfil demográfico y de tratamiento en un centro de referencia. Material y métodos: Se realizó un estudio retrospectivo, descriptivo, observacional, analítico de tipo cohorte en un centro de referencia entre el 01 de Enero de 2005 y el 31 de Diciembre de 2019, se analizaron las variables demográficas y se contrastaron con las variables de desenlace que fueron supervivencia global y período libre de enfermedad. Resultados: Se incluyeron 85 pacientes, la media edad de presentación fue 43.3 años, la etapa clínica al momento de la presentación fue mayormente IIa y IIb abarcando $54.1 \%$, 35 pacientes tuvieron metástasis con etapa clínica IV. El principal sitio de metástasis fue el pulmón en 32 pacientes (37.6\%). De los pacientes, $36(42.3 \%)$ presentaron mortalidad cáncer específica, 35 (41.2\%) fallecieron como consecuencia de la enfermedad metastásica a pulmón u otros órganos y un paciente falleció por extensión directa del tumor en columna cervical. Conclusión: Los factores pronósticos importantes hallados en nuestra serie que influyen en la supervivencia global son: la presencia de metástasis y la localización axial.
\end{abstract}

ABSTRACT. Introduction: Chondrosarcoma is the second most frequent primary malignant bone tumor, is characterized by the formation of a chondroid matrix, is more frequent in advanced ages and its prognosis is directly related to the histological subtype and tumor grade. The objective of this article is to present the demographic and treatment profile in a reference center. Material and methods: A retrospective, descriptive, observational, analytical cohort-type study was conducted in a reference center between January 1, 2005 and December 31, 2019, the demographic variables were analyzed and contrasted with the outcome variables that were overall survival and disease-free period. Results: 85 patients were included, the mean age of presentation was 43.3 years, the clinical stage at the time of presentation was mostly IIa and IIb covering $54.1 \%$. 35 patients had metastases with clinical stage IV. The main site of metastasis was the lung in 32 patients (37.6\%). Thirty-six patients $(42.3 \%)$ had specific mortality. 35 patients $(41.2 \%)$ died as a result of metastatic disease to lung or other organs, one patient died from direct extension of the tumor in the cervical spine. Conclusion: The important prognostic factors found in our series that influence global survival are: the presence of metastases and axial localization.

\section{Nivel de evidencia: III}

\footnotetext{
* Ortopedista Oncólogo. Adscrito, Departamento de Piel y Partes Blandas Instituto Nacional de Cancerología.

^Cirujano Oncólogo. Adscrito, Departamento de Piel y Partes Blandas Instituto Nacional de Cancerología.

$\S$ Ortopedista Oncólogo. Instituto Nacional de Rehabilitación (Ciudad de México).

" Patólogo Oncólogo. Adscrito, Departamento de Piel y Partes Blandas Instituto Nacional de Cancerología.

" Ortopedista oncólogo. Adscrito, Hospital de Alta Especialidad de Veracruz (Veracruz).

** Cirujano Oncólogo. Jefe de Departamento de Piel y Partes Blandas Instituto Nacional de Cancerología.
}

Correspondencia:

MA Clara-Altamirano

Avenida San Fernando Núm. 22, Col. Sección XVI, C.P. 14080, Alcaldía Tlalpan, Ciudad de México.

E-mail: drmiguelclara@gmail.com

Recibido: 09-06-2020. Aceptado: 30-06-2021.

Citar como: Clara-Altamirano MA, García-Ortega DY, Núñez-González M, Caro-Sánchez CHS, Espejo-Sánchez G, Martínez-Said H, et al. Estado actual del condrosarcoma en un centro de referencia. Acta Ortop Mex. 2021; 35(4): 300-304. https://dx.doi.org/10.35366/103307 
Palabras clave: Condrosarcoma, subtipo histológico, metástasis, revisión.

\section{Introducción}

El término condrosarcoma (CS) se usa para describir a un grupo heterogéneo de tumores con características morfológicas y comportamientos clínicos diversos que se caracterizan por la formación de matriz condroide; corresponde al segundo tumor óseo maligno primario más frecuente. ${ }^{1}$

Se reconocen las siguientes variantes de condrosarcoma: CS primario convencional o central, CS del esqueleto de manos y pies o acral, CS de los huesos del macizo craneofacial, CS de las edades infantil y juvenil, CS yuxtacortical o periostal, CS central secundario en la encondromatosis múltiple, en la enfermedad de Ollier y síndrome de Maffucci, CS periférico secundario, CS de células claras, CS desdiferenciado, $\mathrm{CS}$ sinovial, $\mathrm{CS}$ de laringe, $\mathrm{CS}$ mesenquimal y $\mathrm{CS}$ mixoide extraesquelético. Más de $90 \%$ de los CS corresponden al CS convencional, siendo el de bajo grado el que abarca aproximadamente $90 \%$ de la totalidad y se caracteriza por un crecimiento lento, comportamiento indolente y bajo

\begin{tabular}{|c|c|}
\hline Característica & n $(\%)$ \\
\hline Edad & $43.3 \pm 14.23$ \\
\hline \multicolumn{2}{|l|}{ Sexo } \\
\hline Femenino & $39(45.89)$ \\
\hline Masculino & $46(54.1)$ \\
\hline \multicolumn{2}{|l|}{ Subtipo histológico } \\
\hline Convencional & $67(78.8)$ \\
\hline Desdiferenciado & $6(7.1)$ \\
\hline Mixoide & $5(5.9)$ \\
\hline Secundario a tumores benignos & $4(4.7)$ \\
\hline Mesenquimal & $1(1.2)$ \\
\hline Perióstico & $1(1.2)$ \\
\hline Secundario a radioterapia & $1(1.2)$ \\
\hline \multicolumn{2}{|l|}{ Localización } \\
\hline Esqueleto axial & $54(63.5)$ \\
\hline Esqueleto apendicular & $31(36.5)$ \\
\hline \multicolumn{2}{|l|}{ Hueso afectado } \\
\hline Pelvis & $27(31.8)$ \\
\hline Fémur & $11(12.9)$ \\
\hline Húmero & $11(12.9)$ \\
\hline Arcos costales & $10(11.8)$ \\
\hline Escápula & $8(9.4)$ \\
\hline Columna torácica & $5(5.9)$ \\
\hline Columna lumbar & $3(3.5)$ \\
\hline Columna cervical & $1(1.2)$ \\
\hline Sacro & $4(4.7)$ \\
\hline Tibia & $3(3.5)$ \\
\hline Pie & $2(3.5)$ \\
\hline Inicio de síntomas, mediana [rango] & 12 meses [2-180] \\
\hline \multicolumn{2}{|l|}{ Síntoma inicial } \\
\hline Dolor & $54(63.5)$ \\
\hline Tumor & $27(31.8)$ \\
\hline Debilidad & $4(4.7)$ \\
\hline
\end{tabular}

Keywords: Chondrosarcoma, histological subtype, metastasis, revision.

potencial de metástasis. ${ }^{2}$ En contraparte, los CS convencionales de alto grado suelen tener un comportamiento agresivo local y a distancia con altas tasas de recurrencia y menor supervivencia global.

Otras variantes y subtipos histológicos del CS son significativamente menos comunes, entre los que destacan el CS desdiferenciado que frecuentemente exhibe características de osteosarcoma, fibrosarcoma o sarcoma pleomorfo indiferenciado y presenta un comportamiento biológico agresivo. ${ }^{4}$

El CS mesenquimal es un tumor de alto grado que muestra un patrón histológico caracterizado por un componente elevado de células redondas pequeñas indiferenciadas, así como islas de tejido cartilaginoso bien diferenciado con una aberración cromosomal caracterizada por la fusión HEY1NCOA2. Este subtipo histológico tiene un comportamiento muy agresivo con una tendencia a presentar enfermedad metastásica, ya sea al diagnóstico o durante el seguimiento y en contraparte con el resto de los CS, el uso de quimioterapia como parte del manejo integral es imperativo. El CS mixoide extraesquelético es también un tumor de bajo grado que presenta una gran cantidad de degeneración mixoide; se caracteriza por la traslocación $(9 ; 22)(q 22 ; q 12)$ con fusión EWSR1 a NRA 3 (genes que se denominan (CHN, TEC o NOR1). Otros patrones de translocación a NRA3 incluyen TAF15 y TCF12. 1,5,6,7

Los CS son en general resistentes a la quimioterapia, esto se debe a su poca vascularidad que es una característica de la matriz condroide. ${ }^{8}$ El pronóstico del CS se encuentra ligado al grado histológico de la enfermedad, a la presencia o no de metástasis al momento del diagnóstico, la edad de presentación y su localización anatómica. 9,10,11,12,13,14

El objetivo de este artículo es mostrar las variables epidemiológicas y de tratamiento en una cohorte de pacientes con diagnóstico de condrosarcoma manejados en un centro de referencia de México en el período comprendido de 2005 a 2019.

\section{Material y métodos}

Se realizó un estudio retrospectivo, observacional, descriptivo y analítico de tipo cohorte utilizando la base de datos de sarcomas óseos del Departamento de Piel y Partes Blandas del Instituto Nacional de Cancerología de México en el período comprendido entre el 01 de Enero de 2005 y el 31 de Diciembre de 2019 en la que se incluyeron los expedientes de pacientes mayores de 18 años, diagnóstico de condrosarcoma confirmado por estudio histopatológico, seguimiento mínimo de tres meses, con expediente clínico completo; se excluyeron todos aquellos casos con abandono de tratamiento. Se recabaron las variables: edad, sexo, tipo y subtipo histológico, 
Tabla 2: Estadio clínico y tratamiento. $\mathbf{N}=85$.

\begin{tabular}{|cc}
\hline Característica & $\mathrm{n}(\%)$ \\
\hline Estadio clínico Enneking & \\
IA & $3(3.5)$ \\
IB & $14(16.5)$ \\
IIA & $1(1.2)$ \\
IIB & $38(44.7)$ \\
III & $29(34.1)$ \\
Estadio clínico AJCC & \\
IA & $3(3.5)$ \\
IB & $5(5.9)$ \\
IIA & $11(12.9)$ \\
IIB & $35(41.2)$ \\
III & $2(2.4)$ \\
IVA & $25(29.4)$ \\
IVB & $4(4.7)$ \\
Grado histológico & \\
1 & $17(20.0)$ \\
2 & $23(27.0)$ \\
3 & $45(53.0)$ \\
Tratamiento quirúrgico & \\
Sí & $61(71.8)$ \\
No & $24(28.2)$ \\
Cirugía preservadora & \\
Sí & $35(41.2)$ \\
No & $50(58.8)$ \\
Cirugía radical & \\
Sí & $26(30.6)$ \\
No & $59(69.4)$ \\
Tipo de cirugía & \\
Resección amplia & $15(17.7)$ \\
Curetaje + adyuvante + injerto óseo & $2(2.4)$ \\
Curetaje + adyuvante + cemento & $1(1.2)$ \\
Resección amplia + artroplastia & $6(7.0)$ \\
Corpectomía + estabilización anterior & $1(1.2)$ \\
Amputación & $8(9.4)$ \\
Desarticulación & $6(7.0)$ \\
Hemipelvectomía interna & $9(10.6)$ \\
Hemipelvectomía externa & $8(9.4)$ \\
Sacrectomía & $2(2.4)$ \\
Escapulectomía & $3(3.5)$ \\
Radioterapia, n (\%) & \\
Sí & $28(32.9)$ \\
No & $57(67.1)$ \\
AJCC=American Joint Committee on Cancer. & \\
& \\
\hline & \\
\hline &
\end{tabular}

localización anatómica, tipo de tratamiento, recurrencia local o a distancia y supervivencia global.

Se definió como supervivencia global el período comprendido desde el diagnóstico hasta la muerte o última valoración, se consideró recurrencia de la enfermedad como el evento de aparición de la misma después de un período de seis meses de ausencia demostrada por estudios de imagen y valoración clínica. El estadio clínico se evalúo mediante la clasificación de Enneking y la clasificación de la AJCC (American Joint Committee on Cancer, $8^{\text {a }}$. edición). Se realizó un análisis descriptivo de la base de datos obteniendo medidas de tendencia central medias o medianas y de dispersión, rangos o desviaciones estándar según el tipo de distribución observada, se hizo prueba de Kolmogorov-Smirnov para determinar normalidad. Para todas las pruebas estadís- ticas consideramos una $\mathrm{p}<0.05$ como significancia. Para los datos categóricos se utilizó la prueba exacta de Fisher o la prueba de $\chi^{2}$ de Pearson según la distribución de los datos. Para la comparación de medias de las variables cuantitativas se realizó la U de Mann-Whitney o la prueba t de Student según correspondiera. Así también se efectuó análisis inferencial para determinar supervivencia a través del estimador de supervivencia de Kaplan-Meier.

Además, para elaborar un modelo pronóstico se realizó una regresión logística donde la variable dependiente fue la mortalidad y las variables independientes fueron edad, género, estadio clínico, localización, grado tumoral y metástasis. El análisis se efectuó con el paquete de análisis de datos STATA 14.

\section{Resultados}

La base de datos arroja un total de 105 casos, de los cuales sólo 85 cumplieron con los criterios de inclusión, los cuales se tomaron en cuenta para el presente estudio. La distribución de la población fue no paramétrica en casi todas las variables, la única variable cuantitativa con distribución paramétrica fue la edad (Tabla 1).

La etapa clínica al momento de la presentación fue mayormente IIa y IIb abarcando 54.1\%; sin embargo, más de una tercera parte inició con enfermedad metastásica. En la Tabla 2 se resume el tipo de tratamiento que se aplicó en relación directa con las características y estadio clínico. La etapa clínica avanzada y la localización (esqueleto axial vs apendicular) fueron las variables asociadas a mal pronóstico (Figuras 1 y 2).

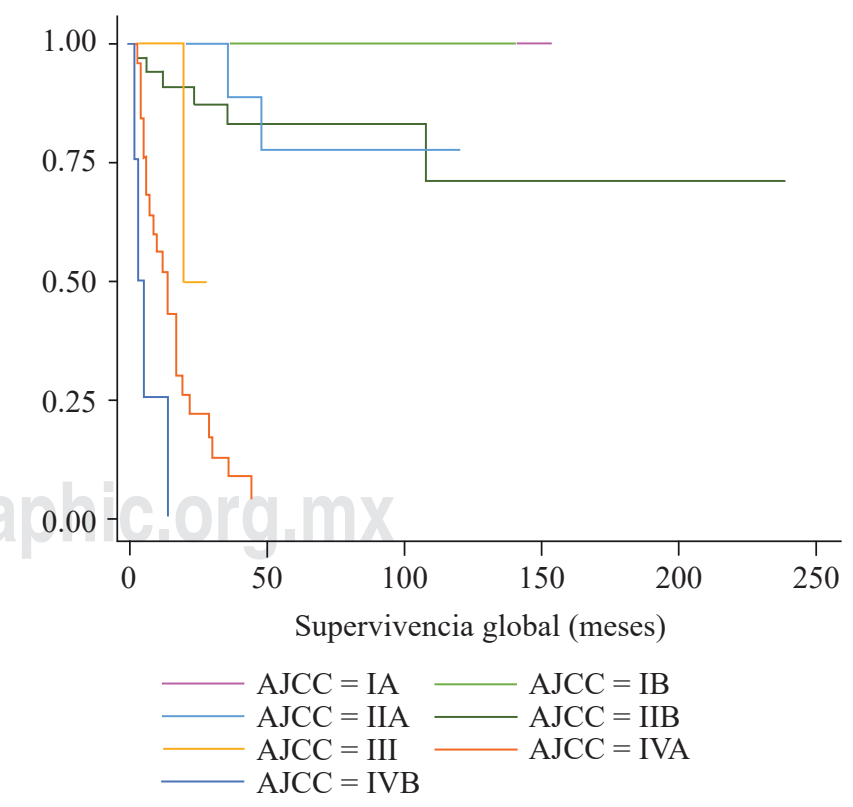

Figura 1: Curva de Kaplan-Meier. Supervivencia global por estadio clínico.

AJCC $=$ American Joint Committee on Cancer. 


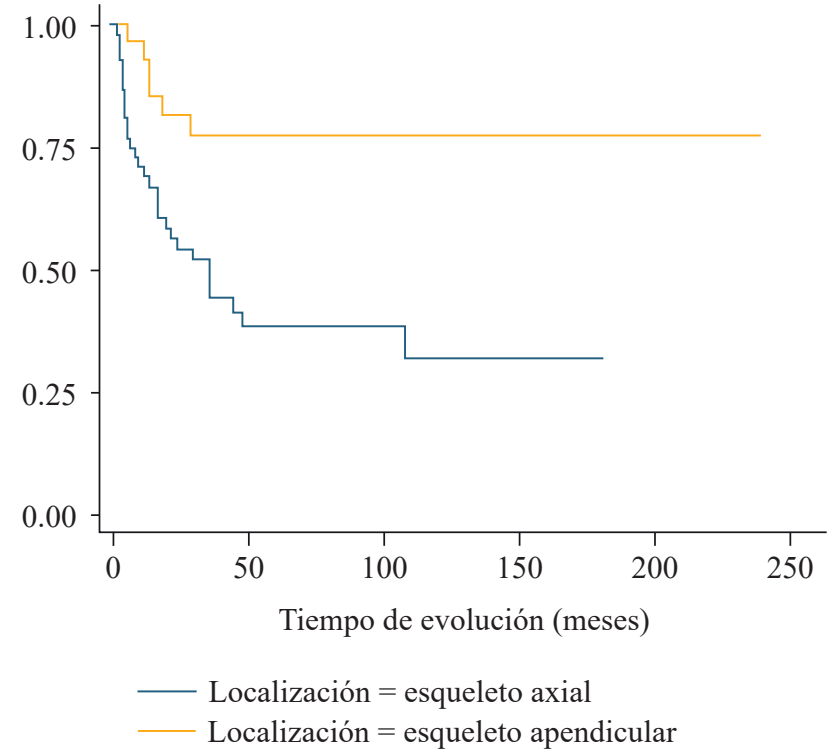

Figura 2: Curva de Kaplan-Meier. Supervivencia global por localización del tumor.

De los pacientes, $35 \%$ presentaron metástasis, de los cuales $26(30.6 \%)$ al momento del diagnóstico y nueve (10.6\%) mostraron progresión de la enfermedad durante el seguimiento. El sitio principal de metástasis fue el pulmón en 32 pacientes $(37.6 \%), 36$ pacientes $(42.3 \%)$ presentaron mortalidad cáncer específica a consecuencia del condrosarcoma. Fallecieron 35 pacientes $(41.2 \%)$ como consecuencia de la enfermedad metastásica a pulmón y otros órganos, un paciente falleció por extensión directa del tumor en columna cervical (Tabla 3).

\section{Discusión}

El CS es un tumor raro que se manifiesta principalmente en pacientes adultos. El género en el cual se presenta con mayor frecuencia es el masculino, como lo pudimos constatar en nuestra cohorte, donde la mayoría de los casos fueron pacientes del sexo masculino (54.1\%). Al igual que lo reportado en la literatura el CS convencional fue el más frecuente presentándose en 67 pacientes $(78.8 \%)$. La mayoría de los pacientes 54 (63.5\%) mostraron localización en esqueleto axial, la pelvis fue el hueso más afectado en 27 pacientes $(31.8 \%)$. El dolor fue el síntoma más frecuente en 54 pacientes $(63.5 \%)$ en contraste con algunas otras publicaciones que reportan la presencia de tumor como el síntoma principal.

En el CS el tratamiento ha cambiado en los últimos años e implica una amplia variedad de tratamientos quirúrgicos. Ejemplo de esto es que el CS de bajo grado actualmente se trata mediante curetaje y colocación de un adyuvante, mientras que el tratamiento del CS grado 2 y 3 se realiza mediante una resección amplia. ${ }^{8,9}$ Esto último también resulta interesante, ya que el margen quirúrgico en los sarcomas de alto grado ha cambiado. ${ }^{15}$ En nuestra serie encontramos CS grado 3 en 45 pacientes, que contrasta con lo reportado en la literatura en la que se observa un mayor porcentaje de pacientes con grado 1, esto quizá de la mano del retraso en la atención y referencia de este grupo de pacientes en los diferentes sistemas de salud en nuestro país y al poco conocimiento de la enfermedad.

Encontramos una relación estadísticamente significativa entre la presencia de metástasis óseas irresecables al momento del diagnóstico y la mortalidad del paciente con una $\mathrm{p}=0.03$, lo que coincide con lo reportado en la literatura al ser uno de los factores pronósticos más importantes en la supervivencia global de los pacientes con un $\mathrm{OR}=25.6$ (IC 95\% $=12.4-37.6$ ) (Figura 1).

El sitio afectado con más frecuencia fue el pulmón en 32 pacientes (37.6\%). Todos los pacientes en estadio clínico IVB de la AJCC (metástasis a pulmones y otros órganos) fallecieron en un período de tres a seis meses (Figura 1).

Encontramos también una relación estadísticamente significativa entre la localización en esqueleto axial y la mortalidad con una $\mathrm{p}=0.001 \mathrm{OR}=3.6(\mathrm{IC} 95 \%=1.4-4.8)(\mathrm{Figu}-$ ra 2). Esto se debe principalmente a que los sarcomas óseos de localización axial suelen tener un tiempo de evolución mayor, si se presentan en la pelvis suelen ser tumores de gran tamaño y las alternativas quirúrgicas en este grupo de pacientes suelen verse muy limitadas. ${ }^{16}$ Debido a la heterogeneidad en la presentación de los pacientes con CS y a la diferencia en el tiempo de evolución antes del diagnóstico no todos los pacientes pudieron ser candidatos a tratamiento quirúrgico. En nuestra revisión, a 61 pacientes $(71.8 \%)$ se les realizó algún tipo de procedimiento quirúrgico, de estos pacientes a tan sólo 35 (41.2\%) se les pudo efectuar cirugía de salvamento de la extremidad, siendo la resección amplia el tratamiento realizado con mayor frecuencia en 15 pacientes (17.7\%); esto difiere de otros reportes donde existe mayor cantidad de cirugías de salvamento de la extremidad; $;^{13,14,15,16}$ sin embargo, la muestra de pacientes que observamos llevaba un largo período de evolución con una mediana de 12 meses y un rango de tres a 180 meses.

Tabla 3: Recurrencia, metástasis y supervivencia global. $\mathbf{N}=\mathbf{8 5}$.

$\begin{array}{lc}\text { Característica } & \mathrm{n}(\%) \\ \text { Metástasis al diagnóstico } & \\ \text { Sí } & 26(30.6) \\ \text { No } & 59(69.4) \\ \text { Metástasis durante el seguimiento } & \\ \text { Sí } & 9(10.6) \\ \text { No } & 76(89.4) \\ \text { Localización } & 32(37.6) \\ \text { Pulmón } & 1(1.2) \\ \text { Otro hueso } & 2(2.4) \\ \text { Otros órganos } & 30[3-238] \\ \text { Supervivencia global, mediana [rango] } & \\ \text { Muerte } & 49(57.7) \\ \text { No } & 36(42.3) \\ \text { Sí } & \end{array}$


De nuestra serie, 28 pacientes $(32.9 \%)$ recibieron tratamiento con radioterapia, la indicación fue en aquellos tumores en esqueleto axial irresecables o en las recurrencias en esqueleto axial irresecables. ${ }^{17}$

Nuestro estudio tiene limitaciones que se relacionan directamente con el carácter retrospectivo y con una gran cantidad de variables; sin embargo, presenta una serie de casos que nos da un panorama global de la enfermedad y por qué no, intentar desarrollar futuras investigaciones. Las diferentes características del CS limitan el tratamiento y condicionan un pronóstico. Por tal motivo, resultará siempre importante la detección oportuna. Proponemos el manejo multidisciplinario para favorecer una mejoría en la supervivencia global de los pacientes.

\section{Conclusión}

El condrosarcoma requiere de un conocimiento profundo de la enfermedad, sus variables pronósticas: edad, localización, subtipo histológico, grado tumoral y etapa clínica pueden ofrecer oportunamente la mejor alternativa quirúrgica.

Las metástasis al momento del diagnóstico y la localización axial son factores pronósticos importantes en la supervivencia global de los pacientes.

Referencias

1. Chow WA. Chondrosarcoma: biology, genetics, and epigenetics. F1000Res. 2018; 7: F1000 Faculty Rev-1826.

2. Gelderblom H, Hogendoorn PC, Dijkstra SD, van Rijswijk CS, Krol AD, Taminiau AH, et al. The clinical approach towards chondrosarcoma. Oncologist. 2008; 13: 320-9.

3. Fromm J, Klein A, Baur-Melnyk A, Knosel T, Lindner L, Birkenmaier C, Dürr HR. Survival and prognostic factors in conventional G1 chondrosarcoma. World Journal of Surgical Oncology. 2019; 17(1): 155.

4. Mankin HJ, Cantley KP, Schiller AL, Lippiello L. The biology of human chondrosarcoma. II. Variation in chemical composition among types and subtypes of benign and malignant cartilage tumors. $J$ Bone Joint Surg Am. 1980; 62(2): 176-88.
5. Zhu GG, Nafa K, Agaram N, Zehir A, Benayed R, Sadowska J, et al. Genomic profiling identifies association of IDH1/IDH2 mutation with longer relapse-free and metastasis-free survival in high-grade chondrosarcoma. Clinical Cancer Research. 2020; 26(2): 419-27.

6. Meijer D, de Jong D, Pansuriya TC, van den Akker BE, Picci P, Szuhai $\mathrm{K}$, et al. Genetic characterization of mesenchymal, clear cell, and dedifferentiated chondrosarcoma. Genes Chromosomes Cancer. 2012; 51(10): 899-909.

7. Hirabayashi Y, Ishida T, Yoshida MA, Kojima T, Ehihara Y, Machinami R, et al. Translocation $(9 ; 22)(\mathrm{g} 22 ;$ q12) A recurrent chromosome abnormality in extraskeletal myxoid chondrosarcoma. Cancer Genetics and Cytogenetics. 1995; 81(1): 33-7.

8. Riedel RF, Larrier N, Dodd L, Kirsch D, Martinez S, Brigman BE. The clinical management of chondrosarcoma. Curr Treat Options Oncol. 2009; 10(1-2): 94-106.

9. Chen X, Yu LJ, Peng HM, Jiang C, Ye CH, Zhu SB, et al. Is intralesional resection suitable for central grade 1 chondrosarcoma: a systematic review and updated meta-analysis. Eur J Surg Oncol. 2017; 43(9): 1718-26.

10. Amer KM, Munn M, Congiusta D, Abraham JA, Basu Mallick A. Survival and prognosis of chondrosarcoma subtypes: SEER database analysis. J Orthop Res. 2020; 38(2): 311-9.

11. Saifuddin A, Sharif B, Oliveira I, Kalus S, Barnett J, Pressney I. The incidence of skip metastases on whole bone MRI in high-grade bone sarcomas. Skeletal Radiol. 2020; 1-10.

12. Verdegaal SH, Bovée JV, Pansuriya TC, Grimer RJ, Ozger H, Jutte PC, et al. Incidence, predictive factors, and prognosis of chondrosarcoma in patients with Ollier disease and Maffucci syndrome: an international multicenter study of 161 patients. Oncologist. 2011; 16(12): 1771.

13. Bergh P, Gunterberg B, Meis-Kindblom JM, Kindblom LG. Prognostic factors and outcome of pelvic, sacral, and spinal chondrosarcomas: a center-based study of 69 cases. Cancer. 2001; 91(7): 1201-12.

14. Nota SP, Braun Y, Schwab JH, van Dijk CN, Bramer JA. The identification of prognostic factors and survival statistics of conventional central chondrosarcoma. Sarcoma. 2015, 2015: 623746.

15. Tsuda Y, Evans S, Stevenson JD, Parry M, Fujiwara T, Laitinen M, Jeys L. Is the width of a surgical margin associated with the outcome of disease in patients with peripheral chondrosarcoma of the pelvis? A multicenter study. Clin Orthop Relat Res. 2019; 477(11): 2432-40.

16. Badaruddin S, Mehtab P, Mubarak MD, Shakeel S, Sohail S. Chondrosarcoma pelvis: a review article. BAOJ Cancer Res Ther. 2019; 5: 062 .

17. Catanzano AA, Kerr DL, Lazarides AL, Dial BL, Lane WO, Blazer $\mathrm{DG}$, et al. Revisiting the role of radiation therapy in chondrosarcoma: a national cancer database study. Sarcoma. 2019; 2019: 4878512. 